\title{
CONCERNING HAUSDORFF MATRICES AND ABSOLUTELY CONVERGENT SEQUENCES ${ }^{1}$
}

\author{
J. P. BRANNEN
}

Introduction. A complex number sequence $B=\left\{b_{n}\right\}$ will be called absolutely convergent $[2$, p. 72$]$ if

$$
\sum_{p}\left|b_{p}-b_{p+1}\right|<\infty .
$$

If the function $g$ is Riemann integrable $[1, p .192]$ on $[0,1]$, the generalized Hausdorff matrix, $H^{\circ}$, determined by $g$ is defined by

$$
H_{n, p}^{0}= \begin{cases}0 & \text { if } p>n \\
\int_{[0,1]}\left(\begin{array}{l}
n \\
p
\end{array}\right) x^{p}(1-x)^{n-p} d g(x) & \text { if } p \leqq n\end{cases}
$$

for $\left.{ }_{p}^{n}\right\}=0,1,2, \cdots$.

This paper is concerned with the determination of those generalized Hausdorff matrices which sum all absolutely convergent sequences. Theorem 2 gives a sufficiency condition stated in terms of the behavior of the points of continuity of the graph of $g$ and Theorems 3 and 4 provide examples which serve to further describe functions which generate such matrices. Theorem 5 gives a necessary and sufficient condition in terms of moment sequences. Theorem 1 gives conditions which are necessary and sufficient for a semi-infinite complex number matrix to sum all absolutely convergent sequences and although the theorem is known [3], a brief proof is included here for completeness since the author does not know of one in the literature.

THEOREM 1. If $A$ is an infinite complex triangular matrix, then the following two statements are equivalent:

(i) If $B$ is an absolutely convergent complex number sequence, then $A \cdot B=T$ converges; and

(ii) (a) there is a number $K$ such that if $n, j$ is a nonnegative integer pair, then

$$
\left|\sum_{p=0}^{i} A_{n, p}\right|<K
$$

Presented to the Society January 22, 1962; received by the editors June 25, 1962 and, in revised form, December 10, 1962.

1 This paper is taken from a thesis written under the direction of Professor H. S. Wall while the author held a National Science Foundation Science Faculty Fellowship. 
(b) the sequence

$$
\left\{\sum_{p=0}^{n} A_{n, p}\right\}_{n=0}^{\infty}
$$

converges, and

(c) for each nonnegative integer $p$, the sequence

$$
\left\{A_{n, p}\right\}_{n=p}^{\infty}
$$

converges.

Proof. Suppose (i) is true. Then (ii)(b) and (ii)(c) follow as consequences of the absolute convergence of $1,1,1, \cdots$, and sequences of the form $0,0,0, \cdots, 0,1,0, \cdots$.

In order to show that (i) implies (ii) (a), it is first established that (i) implies the existence of a number $M$ such that

$$
\left.\left|A_{n, p}\right|<M ; \quad \begin{array}{l}
n \\
p
\end{array}\right\}=0,1,2, \cdots .
$$

Since (i) implies (ii) (c), there is a positive number sequence $C$ such that for each nonnegative integer $p,\left|A_{n, p}\right|\left\langle c_{p} ; n=0,1,2, \ldots\right.$. If (1) is not true, then there is an increasing sequence $N$ of integers and an increasing sequence $P$ of integers such that $p_{0}=0$ and $\left|A_{n_{i}, p_{i}}\right|>4^{i} \sum_{j=0}^{p_{i-1}} c_{j} ; i=1,2,3, \cdots$. Let $B$ be the absolutely convergent sequence

$$
b_{j}= \begin{cases}\frac{1}{2^{i}} & \text { if } j=p_{i}, \quad i=1,2,3, \cdots . \\ 0 & \text { if } j \neq p_{i},\end{cases}
$$

The sequence $T=A \cdot B$ is unbounded since the sequence $C$ is unbounded if (1) is not true;

$$
\begin{aligned}
\left|t_{n_{i}}\right| & =\left|\sum_{p=0}^{n_{i}} A_{n_{i}, p} b_{p}\right|=\left|\sum_{j=1}^{i} \frac{A_{n_{i}, p_{j}}}{2^{j}}\right|>\left|\frac{A_{n_{i}, p_{i}}}{2^{i}}\right|-\sum_{j=0}^{p_{i-1}} \frac{c_{j}}{2^{j}} \\
& >2^{i} \sum_{j=0}^{p_{i-1}} c_{j}\left(1-\frac{1}{2^{i}}\right)>2^{i} \sum_{j=0}^{p_{i-1}} c_{j} .
\end{aligned}
$$

This contradicts the assumption that (i) is true, hence (1) is true.

If (ii)(a) is not true, then for each $c>0$ there is a nonnegative integer pair $n, j$ such that $\left|\sum_{p=0}^{j} A_{n, p}\right|>c$. Let $M$ be the number 
given in (1); and for each nonnegative integer $k$, let $n_{k}, j_{k}$ be an integer pair such that

(2) $\left|\sum_{p=0}^{j_{0}} A_{n_{0}, p}\right|>4 M$ and $\left|\sum_{p=0}^{j_{k}} A_{n_{k}, p}\right|>4^{k+1} M 2 n_{k-1} ; \quad k \geqq 1$.

Let $B$ be the absolutely convergent sequence such that for each nonnegative integer $p$,

$$
b_{p}=\left\{\begin{array}{cl}
1 & \text { if } p \leqq j_{0} . \\
\frac{1}{2^{k+1}} & \text { if } n_{k} \leqq p \leqq j_{k+1} ; \quad k=0,1,2, \cdots . \\
0 & \text { if } j_{k}<p<n_{k} .
\end{array}\right.
$$

Consider the sequence $T=A \cdot B$. Using (1)

$$
\left|t_{n_{k}}\right| \geqq \frac{1}{2^{k}}\left|\sum_{p=n_{k-1}}^{j_{k}} A_{n_{k}, p}\right|-M n_{k-1} ; \quad k=1,2,3, \cdots ;
$$

and from (1) and (2) it follows that

$$
\left|\sum_{p=n_{k-1}}^{i_{k}} A_{n_{k}, p}\right|>4^{k} M n_{k-1} .
$$

Therefore,

$$
\left|t_{n_{k}}\right|>2^{k} M n_{k-1}-M n_{k-1}>M n_{k-1} 2^{k-1},
$$

from which it follows that $T$ is unbounded, contrary to (i) being true. Thus, (i) implies (ii).

Suppose (ii) is true and $B$ is an absolutely convergent sequence. If $C$ is a constant term sequence and $D$ is the sequence such that for each nonnegative integer $n, d_{n}=b_{n}-c$, then $A \cdot B=A \cdot C+A \cdot D$. Therefore, we shall suppose that $B$ has limit 0 .

There is a number $K^{\prime}$ such that

$$
K^{\prime}>\left\{\begin{array}{l}
\left|b_{0}\right|+\sum_{p=0}^{\infty}\left|b_{p}-b_{p+1}\right| \\
\left.\left|\sum_{p=0}^{j} A_{n, p}\right|, \begin{array}{c}
n \\
j
\end{array}\right\}=0,1,2, \cdots, \\
\left.\left|A_{n, p}\right|, \begin{array}{l}
n \\
p
\end{array}\right\}=0,1,2, \cdots, \text { and } \\
\left|b_{n}\right|, \quad n=0,1,2, \cdots .
\end{array}\right.
$$


If $c>0$, let $N^{\prime}$ be a positive integer such that if $n>N^{\prime}$, then

$$
\sum_{p=n}^{\infty}\left|b_{p}-b_{p+1}\right|<\frac{c}{32 K^{\prime}} \text { and }\left|b_{n}\right|<\frac{c}{16 K^{\prime}} .
$$

For each nonnegative integer $m \leqq N^{\prime}$, let $j_{m}$ be a positive integer such that if $n>j_{m}$ and $k$ is a positive integer, then

$$
\left|A_{n, m}-A_{n+k, m}\right|<\frac{c}{16 N^{\prime} K^{\prime} 2^{m}} .
$$

Let

$$
N=\sum_{m=0}^{N^{\prime}} j_{m}
$$

If $n>N$ and $k=1,2,3, \cdots$, then

$$
\left|t_{n}-t_{n+k}\right|=\left|\sum_{p=0}^{n} A_{n, p} b_{p}-\sum_{p=0}^{n+k} A_{n+k, p} b_{p}\right| .
$$

By use of the triangle inequality and summation by parts, it follows that the right side of (5) is equal to or less than

$$
\begin{aligned}
& \sum_{j=0}^{n-1}\left(\left|b_{j}-b_{j+1}\right| \sum_{p=0}^{j}\left|A_{n, p}-A_{n+k, p}\right|\right)+\left|b_{n}\right|\left|\sum_{p=0}^{n} A_{n, p}\right| \\
& \quad+\left|b_{n+k}\right|\left|\sum_{p=0}^{n+k} A_{n+k, p}\right|+\sum_{j=n}^{n+k-1}\left(\left|b_{j}-b_{j+1}\right|\left|\sum_{p=0}^{j} A_{n, p}\right|\right) .
\end{aligned}
$$

It follows from (3), (4), and (6) that $\left|t_{n}-t_{n+k}\right|<c$. Therefore, (ii) implies (i).

Definition 1. $R[a, b]$ is the function set to which $g$ belongs if and only if $g$ is Riemann integrable on $[a, b]$.

Definition 2. If $n, p, p \leqq n$, is a nonnegative integer pair, then

$$
f_{n, p}(x)=\left(\begin{array}{l}
n \\
p
\end{array}\right) x^{p}(1-x)^{n-p} .
$$

If $g$ is in $R[0,1]$, then $\int_{[0,1]} f_{n, p} d g$ exists.

Proofs for the following lemmas are omitted.

\section{Lemma 1. Suppose}

(a) $g$ is in $R[0,1]$ and

(b) $K$ is a number such that if $t$ is in $[0,1]$ and $g$ is continuous at $t$, then $|g(t)|<K$; 


$$
\left|\int_{[0,1]} g d f_{n, p}\right| \leqq K V_{[0,1]} f_{n, p},
$$

where $V_{[0,1]} f_{n, p}$ denotes the variation of $f_{n, p}$ on $[0,1]$.

LEMMA 2. If $t$ is in the sect $(0,1]$ and $p$ is a nonnegative integer, then $f_{n, p}(x) \rightarrow 0$ as $n \rightarrow \infty$ uniformly on $[t, 1]$.

LEMMA 3. If $n, j, j \leqq n$, is a nonnegative integer pair, then

$$
\sum_{p=0}^{j} f_{n, p}(x) \text { is nonincreasing on }[0,1] \text {. }
$$

LEMMA 4. If $p$ is a nonnegative integer and $t$ is a number in $(0,1]$, then there is a number $K$ such that if $x$ is in $[t, 1]$ and $n=p, p+1$, $p+2, \cdots$, then

$$
\left|f_{n, p}^{\prime}(x)\right|<K
$$

Definition 3. If $g$ is in $R[0,1], M_{g}$ is the point set to which the point $p$ with abscissa $x$ belongs if and only if $x$ is in $[0,1]$ and $g$ is continuous at $x$.

THEOREM 2. If $g$ is in $R[0,1]$ and $M_{g}$ has only one limit point on the $Y$-axis, then $H^{\circ}$ satisfies the conditions of Theorem 1.

Proof. Integration by parts plus Lemma 3 shows that $H^{\circ}$ satisfies (ii) (a). That $H^{\circ}$ satisfies (ii)(b) follows from the fact that for each nonnegative integer $n$

$$
\sum_{p=0}^{n} H_{n, p}^{g}=\int_{[0,1]} 1 d g .
$$

In order to show that $H^{\sigma}$ satisfies (ii)(c), we suppose that $p$ is a nonnegative integer and $c>0$. Let $L$ be a number such that if $x$ is in $[0,1]$, then

$$
|g(x)|<L .^{2}
$$

Let $(0, a)$ be the limit point of $M_{0}$ which lies on the $Y$-axis. There is a $t>0$ such that if $x$ is in $[0, t]$ and $g$ is continuous at $x$, then

$$
|g(x)-a|<\frac{c}{L+1}
$$

There is a positive integer $N$ such that if $n>N$, then

${ }^{2}$ From the definition of the $R-S$ integral used here, it follows that if $g$ is in $R[0,1]$, then $g$ is bounded. 
(9)

$$
f_{n, p}(x) \text { is decreasing on }[t, 1] \text { and } f_{n, p}(t)<\frac{c}{L+1} \text {. }
$$

Suppose $p=0$. Integration by parts shows that

$$
\int_{[0, t]} f_{n, 0} d g=f_{n, 0}(t) g(t)-g(0)-a f_{n, 0}(t)+a-\int_{[0, t]}(g-a) d f_{n, 0}
$$

It follows from (7) and (9) that

$$
\left|a f_{n, 0}(t)\right|<c \text { and }\left|g(t) f_{n, 0}(t)\right|<c .
$$

Also, from Lemma 1 and (8) it follows that

$$
\left|\int_{[0, t]}(g-a) d f_{n, 0}\right|<c V_{[0,1]} f_{n, 0}=c .
$$

Integration by parts plus (9) shows that

$$
\left|\int_{[t, 1]} f_{n, 0} d g\right|<\left|g(t) f_{n, 0}(t)\right|+L V_{[t, 1]} f_{n, 0}<2 c .
$$

It follows, by use of (10), (11), (12), and (13), that

$$
\left|\int_{[0,1]} f_{n, 0} d g-[a-g(0)]\right|<5 c .
$$

If $p>0$, an argument similar to that above shows that

$$
\int_{[0,1]} f_{n, p} d g \rightarrow 0 \text { as } n \rightarrow \infty
$$

Therefore, $H^{\sigma}$ satisfies (ii)(c).

THEOREM 3. There is in $R[0,1]$ a function $h$ such that $M_{h}$ has two limit points on the $Y$-axis and $H^{h}$ satisfies the conditions of Theorem 1.

Proof. Let $T$ be a decreasing sequence which lies in $(0,1)$ and has 0 as its limit. By Lemma 4, there is a number sequence $K$ such that if $p$ is a nonnegative integer and $x$ is in $\left[t_{p}, 1\right]$, then for $j=0,1,2$, $\cdots, p$ and $n=j, j+1, j+2, \cdots$

$$
\left|f_{n, j}^{\prime}(x)\right|<k_{p}
$$

Let $S$ be a sequence such that for $p=0,1,2, \ldots$

$$
s_{p+1}<t_{p}<s_{n}<1
$$

and 


$$
\left(s_{p}-t_{p}\right) k_{p}<\frac{1}{2^{p}} .
$$

Let

$$
h(x)= \begin{cases}0 & \text { if } s_{0} \leqq x \leqq 1 \\ 0 & \text { if } s_{p+1} \leqq x \leqq t_{p}, p=0,1,2, \cdots, \\ 1 & \text { if } t_{p}<x<s_{p}, p=0,1,2, \cdots, \\ 0 & \text { if } x=0\end{cases}
$$

$h$ is in $R[0,1]$ and it was established in Theorem 2 that this alone is sufficient for $H^{h}$ to satisfy (ii) (a) and (ii)(b) of Theorem 1.

In order to show that $H^{h}$ satisfies (ii)(c), we suppose that $p$ is a nonnegative integer and that $c>0$. There is a positive integer $N>p$ such that if $n>N$, then

$$
\sum_{j=n}^{\infty} \frac{1}{2^{i}}<\frac{c}{8} .
$$

There is a positive integer $N^{\prime}$ such that if $n>N^{\prime}$, then

$$
f_{n, p} \text { is decreasing on }\left[t_{N}, 1\right] \text { and }
$$

$$
\left|f_{n, p}\left(t_{N}\right)\right|<\frac{c}{8} .
$$

Let $N^{\prime \prime}=N+N^{\prime}$.

Suppose $n>N^{\prime \prime}$.

$$
\begin{aligned}
& \left|\int_{[0,1]} f_{n, p} d h\right| \\
& \quad=\left|\int_{\left(0, t_{N}\right]} f_{n, p} d h+f_{n, p}(1) h(1)-f_{n, p}\left(t_{N}\right) h\left(t_{N}\right)-\int_{\left[t_{N}, 1\right]} h d f_{n, p}\right| .
\end{aligned}
$$

But, $h(1)=0, h\left(t_{N}\right)=0$ and it follows from (17) that

$$
\left|\int_{\left[t_{N}, 1\right]} h d f_{n, p}\right|<\frac{c}{8} .
$$

Therefore, the right side of (18) is less than

$$
\left|\int_{\left[0, t_{N}\right]} f_{n, p} d h\right|+\frac{c}{4} .
$$

There is an integer $M>N$ such that 


$$
V_{\left[0, t_{M}\right]} f_{n, p}<\frac{c}{4}
$$

Since $h(0)=0$ and $h\left(t_{M}\right)=0$, integration by parts plus the triangle inequality shows that

$$
\left|\int_{\left[0, t_{N}\right]} f_{n, p} d h\right| \leqq\left|\int_{\left[0, t_{M}\right]} h d f_{n, p}\right|+\left|\int_{\left[t_{M}, t_{N}\right]} f_{n, p} d h\right| .
$$

It follows from (20) that

$$
\left|\int_{\left[0, t_{M}\right]} h d f_{n, p}\right|<\frac{c}{4} .
$$

Since $h$ is a step function on $\left[t_{M}, t_{N}\right]$, the triangle inequality shows that

$$
\left|\int_{\left[t_{M}, t_{N}\right]} f_{n, p} d h\right| \leqq \sum_{j=0}^{M-N}\left|f_{n, p}\left(t_{M-j}\right)-f_{n, p}\left(s_{M-j}\right)\right| .
$$

$N$ is greater than $p$. Therefore, if $t$ is in $\left[t_{M-j}, t_{N}\right]$, then $\left|f_{n, p}^{\prime}(t)\right|<k_{M-j}$ so that

$$
\left|f_{n, p}\left(t_{M-j}\right)-f_{n, p}\left(s_{M-j}\right)\right|<k_{M-j}\left|s_{M-j}-t_{M-j}\right|
$$

From (15),

$$
\sum_{j=0}^{M-N} k_{M-j}\left|s_{M-j}-t_{M-j}\right|<\sum_{j=0}^{M-N} \frac{1}{2^{M-j}},
$$

which by (16) is less than $c / 8$ so that

$$
\left|\int_{\left[t_{M}, t_{N}\right]} f_{n, p} d h\right|<\frac{c}{8} .
$$

It follows then, from (18), (19), (21), (22), and (23), that

$$
\left|\int_{[0,1]} f_{n, p} d h\right|<c .
$$

Therefore, $H^{h}$ satisfies (ii)(c).

TheOREM 4. There is a function $g$ in $R[0,1]$ such that $M_{0}$ has two limit points on the $Y$-axis and $H^{\circ}$ does not satisfy the conditions of Theorem 1.

Proof. It is sufficient to find a $g$ in $R[0,1]$ such that 


$$
\left\{\int_{[0,1]} f_{n, 1} d g\right\}_{n=1}^{\infty}
$$

diverges.

For each positive integer $n$ and each $x$ in $[0,1]$,

$$
f_{n, 1}(x) \leqq f_{n, 1}\left(\frac{1}{n}\right)=\left(1-\frac{1}{n}\right)^{n-1} \rightarrow e^{-1} \text {. }
$$

There exist positive integer sequences $N$ and $J$ such that

$$
\begin{gathered}
n_{p}<j_{p}<n_{p+1}, \quad p=1,2,3, \cdots, \\
f_{j_{p}, 1}\left(\frac{1}{n_{p+1}}\right)<\frac{e^{-1}}{8}
\end{gathered}
$$

and

$$
f_{j_{p}, 1}\left(\frac{1}{n_{p}}\right)<\frac{e^{-1}}{8}
$$

It follows from these conditions that

$$
\begin{aligned}
& f_{j_{p}, 1} \text { is increasing on }\left[0, \frac{1}{n_{p+1}}\right] \\
& \text { and is decreasing on }\left[\frac{1}{n_{p}}, 1\right] .
\end{aligned}
$$

Let

$$
g(x)= \begin{cases}0 & \text { if } \frac{1}{j_{0}} \leqq x \leqq 1 \\ 1 & \text { if } \frac{1}{j_{2 p+1}}<x<\frac{1}{j_{2 p}}, p=0,1,2, \cdots, \\ 0 & \text { if } \frac{1}{j_{2 p}} \leqq x \leqq \frac{1}{j_{2 p-1}}, p=1,2,3, \cdots, \\ 0 & \text { if } x=0 .\end{cases}
$$

Suppose $p$ is a positive integer.

$$
\begin{aligned}
\int_{[0,1]} f_{j_{p}, 1} d g= & -\int_{\left[0,1 / n_{p+1}\right]} g d f_{j_{p}, 1}-\int_{\left[1 / n_{p+1} 1 / n_{n}\right]} g d f_{j_{p}, 1} \\
& -\int_{[1 / n, 1]} g d f_{j_{p}, 1} .
\end{aligned}
$$


From (24) it follows that

$$
\left.\begin{array}{l}
\left|\int_{\left[0,1 / n_{p+1}\right]} g d f_{j_{p}, 1}\right| \\
\left|\int_{\left[1 / n_{p}, 1\right]} g d f_{j_{p}, 1}\right|
\end{array}\right\}<e^{-1 / 8 .}
$$

Also,

$$
\begin{aligned}
& -\int_{\left[1 / n_{p+1}, 1 / n_{p}\right]} g d f_{j_{p}, 1} \\
& \quad=+f_{j_{p}, 1}\left(1 / n_{p+1}\right) g\left(1 / n_{p+1}\right)-f_{j_{p}, 1}\left(1 / n_{p}\right) g\left(1 / n_{p}\right) \pm f_{j_{p}, 1}\left(1 / j_{p}\right),
\end{aligned}
$$

with the sign of the last term negative if $p$ is even and positive if $p$ is odd. Therefore, if $p$ is even the right side of (25) is less than $-e^{-1}+e^{-1} / 4$ and if $p$ is odd the right side of (25) is greater than $e^{-1}-e^{-1} / 4$ so that $\int_{[0,1]} f_{j_{p}, 1} d g$ is less than $-e^{-1 / 2}$ if $p$ is even and is greater than $e^{-1 / 2}$ if $p$ is odd. Therefore, the sequence

$$
\left\{\int_{[0,1]} f_{n, 1} d g\right\}_{n=1}^{\infty}
$$

contains a divergent subsequence which shows that $H^{o}$ does not satisfy (ii) (c).

TheOREM 5. If $g$ is in $R[0,1]$ and $C$ is the moment sequence determined by $\mathrm{g}$, then $H^{\circ}$ satisfies the conditions of Theorem 1 if and only if for each nonnegative integer $p$ the sequence

$$
\left\{\left(\begin{array}{l}
n \\
p
\end{array}\right) \Delta^{n-p} c_{p}\right\}_{n=p}^{\infty}
$$

converges.

Since the conclusion follows readily from the conditions of statement (ii) Theorem 1, the proof is omitted.

\section{REFERENCES}

1. T. M. Apostol, Mathematical analysis, Addison-Wesley, Reading, Mass., 1957.

2. K. Knopp, Infinite sequences and series, Dover, New York, 1956.

3. B. Kuttner, On discontinuous Riesz means of type n, J. London Math. Soc. 37 (1962), 354-364.

UNIVERSITY OF TEXAS AND

Sandia Corporation 